\title{
Carotid access in Transcatheter Aortic Valve Implantation - an alternative to the gold standard. A single-center experience
}

\author{
Aleksandra Stańska1 ${ }^{1}$, Aleksandra Wierzbowska1, Radosław \\ Targoński' ${ }^{1}$, Wojciech Karolak$^{1} \odot$, Agnieszka Mickiewicz² ${ }^{\oplus}$, \\ Miłosz Jaguszewski² ${ }^{\circledR}$, Dariusz Jagielak ${ }^{1}$
}

${ }^{1}$ Department of Cardiac and Vascular Surgery, Medical University of Gdańsk, Poland

${ }^{2} 1$ st Department of Cardiology, Medical University of Gdańsk, Poland

\begin{abstract}
Background: Transfemoral access is regarded as the TAVI gold standard for the transcatheter aortic valve implantation (TAVI) procedure. However, other options for vascular access have developed in the last few years. Access via the carotid artery is one such alternative. Materials and methods: The study included 9 elderly patients who underwent transcarotid TAVI procedure at the Cardiac and Vascular Surgery Department of the Medical University of Gdańsk. Procedures were performed by a local Heart Team in a hybrid operating room under general anesthesia. Data was collected before the implantation and at discharge. Results: The mean patients' age was 81 years of age (64-88) and the mean logistic EuroSCORE was 10.8 (7-16). Implantations were performed with $100 \%$ device success rate. Intra-operative valve-in-valve procedure was performed in one patient; there were no access-related and valve-related complications during the surgery. Post-procedural complications included minor bleeding, hematoma and pneumothorax. Echocardiographic parameters were significantly improving after the procedure. The mean hospital stay was 5 days (2-7 days). Conclusions: Transcatheter aortic valve implantation via the carotid artery appears to be safe and effective alternative to standard TAVI vascular access.
\end{abstract}

Keywords: transcatheter aortic valve implantation - aortic stenosis · carotid artery · elderly

\section{Citation}

Stańska A, Wierzbowska A, Targoński R, Karolak W, Mickiewicz A, Jaguszewski M, Jagielak D. Carotid access in Transcatheter Aortic Valve Implantation - an alternative to the gold standard. A single-center experience.

Eur J TransI Clin Med. 2019;2(2):80-84.

DOI: $10.31373 /$ ejtcm/116213

Corresponding author:

Aleksandra Stańska, Department of Cardiac and Vascular Surgery, Medical University of Gdańsk, Poland

e-mail: astanska@gumed.edu.pl

No external funds.

Available online: www.ejtcm.gumed.edu.pl

Copyright $₫$ Medical University of Gdańsk

This is Open Access article distributed under the terms of the Creative Commons Attribution-ShareAlike 4.0 International. 


\section{Introduction}

Due to an aging population, the number of patients with symptomatic aortic stenosis (AS) continues to grow. Since transcatheter aortic valve implantation's (TAVI) introduction, it has become a well-established procedure for the treatment of severe symptomatic aortic stenosis, especially in the elderly high-risk patients' population. Currently TAVI is globally recognized as an effective and safe alternative to open heart surgery [1-3].

Transcatheter aortic valve implantation via transfemoral access is regarded as the TAVI gold standard [4]. Approximately $70 \%$ of TAVI procedures are performed using this approach [5]. However, a large number of patients with severe peripheral atherosclerosis and calcified vessels, significant descending aortic disease or physiological abnormalities of the vasculature are considered for alternative approaches [6-7]. As the TAVI experience in the last few years has significantly increased, so have the options for vascular access [2] In cases of difficult anatomy of the femoral-iliac-axis the analysis of risk-to-benefit ratio suggests looking for alternative pathways. Considering the reported high mortality rate of the transthoracic approaches [8], transcarotid access was recently suggested as one the alternative options.

The first case of transcatheter aortic valve implantation via left carotid artery was reported in 2010 and was described as a last resort, when other approaches were unavailable [9]. Furthermore, it should be performed only after a rigorous cerebral arterial evaluation. However, it has been recently reported that transcarotid approach has very similar outcomes as the transfemoral one in regard to mortality and morbidity [10-11]. Moreover, Overtchouk, et al. proved that the frequency of cerebrovascular events after transcarotid TAVI was similar to transfemoral approach [1213]. Considering this, transcarotid access seems to be a very attractive and effective alternative to the transfemoral gold standard.

The aim of this article is to present short-term results of nine transcarotid transcatheter aortic valve implantation procedures performed at the Cardiac and Vascular Surgery Department of the Medical University of Gdańsk from December 2016 to December 2017.

\section{Materials and methods}

\section{Patients and study design}

The study included nine patients with severe, symptomatic aortic stenosis, who underwent transcarotid transcatheter aortic implantation procedure. All the patients were disqualified from transfemoral or other alternative TAVI approaches, due to the extensive calcification of the ascending aorta and aortic arch (porcelain aorta) or poor peripheral access.

Computed tomography angiography and Carotid Doppler was performed on every patient in order to assess the possibility of transcarotid pathway.

All data was collected during a hospital stay - before the procedure and at discharge.

\section{Transcarotid TAVI procedure}

All TAVI procedures were performed by a local Heart Team in a hybrid operating room equipped with a heart-lung machine on stand-by. Implantations were performed under general anesthesia. An approximately $5 \mathrm{~cm}$ vertical incision above the left clavicle was made in order to expose the carotid artery.

The position of the prosthesis was confirmed using a contrast medium. After the implantation, a further bolus of contrast medium was given to assess the position of the valve and to estimate the paravalvular leak (PVL) and coronary ostia. After estimating the shape of the valve and possible PVL, the decision whether to carry out post-dilatation was made.

\section{Results}

Patients' characteristics and parameters at baseline are presented in Table 1. The variables are presented as frequencies (percentages) and means. The mean procedure time was $M=59$ minutes (45-120 minutes) and the mean carotid artery closure time was $M=9,5$ minutes (4-20 minutes). One patient required pre-dilatation, whereas post-dilatation was performed in two

Table 1. Preoperative characteristics and parameters $(n=9)$

\begin{tabular}{|c|c|}
\hline Age (years) & $81(64-88)$ \\
\hline $\begin{array}{c}\text { Male } \\
\text { Female }\end{array}$ & $7(77.8 \%)$ \\
$2(22.2 \%)$
\end{tabular}




\begin{tabular}{|c|c|}
\hline Pulmonary hypertension & $1(11.1 \%)$ \\
\hline Diabetes mellitus & $2(22.2 \%)$ \\
\hline Renal dysfunction & $2(22.2 \%)$ \\
\hline Coronary artery disease & $9(100 \%)$ \\
\hline $\begin{array}{l}\text { Chronic obstructive } \\
\text { pulmonary disease }\end{array}$ & $3(33.3 \%)$ \\
\hline Previous coronary surgery & $1(11.1 \%)$ \\
\hline $\begin{array}{l}\text { Previous coronary } \\
\text { angioplasty }\end{array}$ & $8(88.9 \%)$ \\
\hline $\begin{array}{l}\text { Previous stroke } \\
\text { or transient ischemic attack }\end{array}$ & $1(11.1 \%)$ \\
\hline Atrial fibrillation & $5(55.6 \%)$ \\
\hline Peripheral arterial disease & $5(55.6 \%)$ \\
\hline \multicolumn{2}{|l|}{ NYHA classification } \\
\hline $\begin{array}{c}\text { I } \\
\text { II } \\
\text { III } \\
\text { IV }\end{array}$ & $\begin{array}{c}- \\
3(33.3 \%) \\
6(66.7 \%) \\
-\end{array}$ \\
\hline $\begin{array}{l}\text { Conduction disorders } \\
\text { (LBBB, RBBB, AVB) }\end{array}$ & $0(0 \%)$ \\
\hline $\begin{array}{l}\text { Pre-existing permanent } \\
\text { pacemaker }\end{array}$ & $1(11.1 \%)$ \\
\hline $\begin{array}{l}\text { Aortic valve insufficiency } \\
\qquad(\geq \text { mild })\end{array}$ & $1(11.1 \%)$ \\
\hline $\begin{array}{l}\text { Mitral valve insufficiency } \\
\qquad(\geq \text { mild })\end{array}$ & $9(100 \%)$ \\
\hline
\end{tabular}

AVB - atrio-ventricular block, LBBB - left bundle branch block, NYHA - New York Heart Association functional class, RBBB - right bundle branch block

cases. Intra-operative valve-in-valve procedure (Fig. 1) was performed in one patient due to too low implantation. There were no vascular access-site complications nor valve-related complications. Procedures were performed with $100 \%$ device success rate.

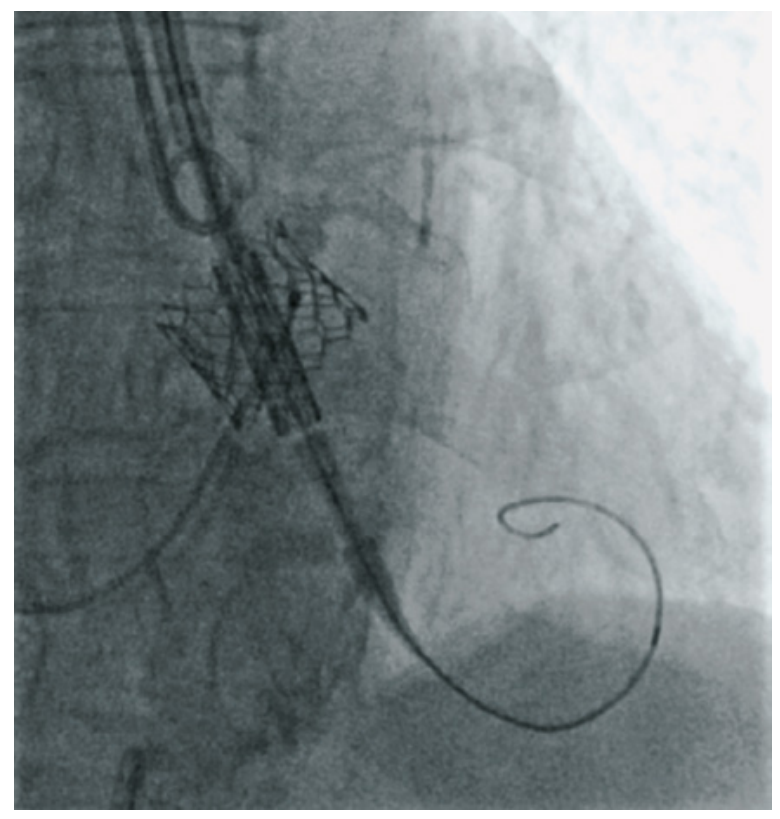

Figure 1. Intra-operative valve-in-valve procedure

Evolut R valve (Medtronic) was implanted in seven patients (78\%) and SAPIEN 3 transcatheter heart valve (Edwards Lifesciences) was implanted in two patients (22\%); six (67\%) $29 \mathrm{~mm}$ sized valves were used, two (22\%) $26 \mathrm{~mm}$ and one (11\%) in $23 \mathrm{~mm}$ size.

Post-procedural complications included minor bleeding, hematoma which required surgical debridement and right-sided pneumothorax. Patients were discharged from the ward after approximately $M=4$ days (2-7 days). The echocardiographic assessment at discharge confirmed good hemodynamic profile (Table 2 ).

Table 2. Echocardiographic aortic valve function before the procedure and at discharge

\begin{tabular}{|c|c|c|}
\hline $\begin{array}{c}\text { Peak gradient } \\
(\mathrm{mmHg})\end{array}$ & $77.7(67-95)$ & $19.4(13-22)$ \\
\hline $\begin{array}{c}\text { Mean gradient } \\
(\mathrm{mmHg})\end{array}$ & $46.8(41-57)$ & $11.1(7-14)$ \\
\hline $\begin{array}{c}\text { Peak aortic } \\
\text { velocity }(\mathrm{m} / \mathrm{s})\end{array}$ & $4.4(4.1-4.8)$ & $2.3(2.2-2.4)$ \\
\hline $\begin{array}{c}\text { Effective orifice } \\
\text { area }\left(\mathrm{cm}^{2}\right)\end{array}$ & $.78(0.5-0.9)$ & $1.8(1.5-2)$ \\
\hline
\end{tabular}




\section{Discussion}

The main aim of the study conducted at the Cardiac and Vascular Surgery Department of the Medical University of Gdańsk was to evaluate the safety and hemodynamic parameters of the transcarotid TAVI procedure.

A high rate of procedural success and a small number of complications are presented. Furthermore, echocardiographic aortic valve function after the procedure was quite satisfactory. No patients experienced any neurological injury during, nor after the procedure.

It is well-known that the manipulation of carotid arteries may increase the risk of neurological injury, however the preliminary data suggest that patients undergoing TAVI procedure via transcarotid access are not exposed to severe neurological impairment. The key to success is probably thorough diagnostic process, including Carotid Doppler and computed tomography angiography. Assessing calcification and diameter of left carotid artery should be the most important part of the pre-operative qualification. In case of any doubts, it may be more adequate to choose non-vascular type of access.

The most important advantage of transcarotid access is a direct and a significantly shorter route to the aortic valve from the entry. The additional benefit is an improved movement precision during catheter delivery.

As in the case of every TAVI approach, there are also certain disadvantages of particular access. The transcarotid procedure is usually performed under general anesthesia and is more invasive in comparison to the transfemoral approach, which is conducted under local anesthesia. Assuming that TAVI should be a less-invasive procedure, the process of evaluation ought to be very specific and, in case of transcarotid access, exclude high-risk patients with a large number of comorbidities. Transcarotid pathway requires larger surgical intervention (Fig. 2) and hence wound-related complications may occur, thus may not be suitable for all patients disqualified from the traditional transfemoral access.

There are some limitations of the study. First of all, it is a single-center experience. Secondly, a small sam-

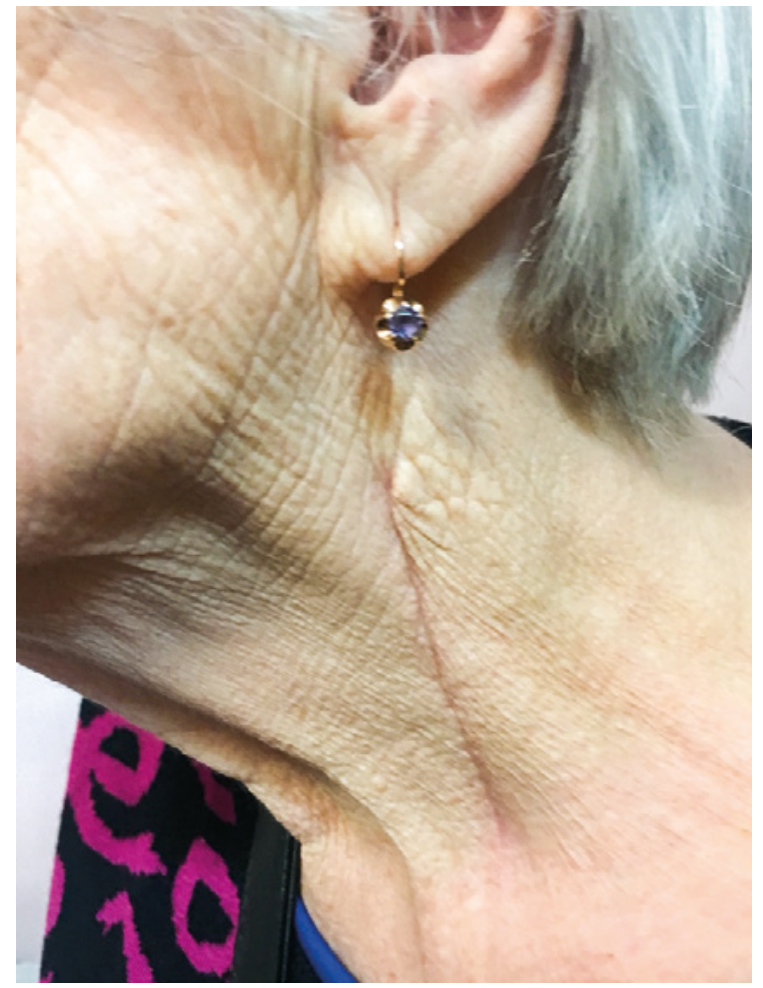

Figure 2. Transcarotid pathway requires larger surgical intervention

ple of patients was included. The analysis of a larger sample may offer slightly different results. Unfortunately, transcarotid access is still not considered as a common alternative to transfemoral approach, so the number of patients' undergoing TAVI via this access is quite small. Considering promising results this approach should be taken into consideration as a second- or third-choice access to the established gold standard.

\section{Conclusions}

The preliminary data suggest that transcarotid transcatheter aortic valve implantation may be a safe and up-and-coming alternative to other types of vascular access. However, this requires further study and multi-center experience.

\section{References}

1. Liu Z, Kidney E, Bem D, Bramley G, Bayliss S, de Belder MA, et al. Transcatheter aortic valve implantation for aortic stenosis in high surgical risk patients: A systematic review and meta-analysis. Paul T, editor. PLoS One [Internet]. 2018 May 10 [cited 2019 Dec 31];13(5):e0196877. Available from: https://dx.plos.org/10.1371/journal.pone.0196877

2. Kanwar A, Thaden JJ, Nkomo VT. Management of patients with aortic valve stenosis. Mayo Clin Proc [Internet]. $2018 \mathrm{Apr}$ [cited 2019 Dec 31];93(4):488-508. Available from: https://linkinghub.elsevier.com/retrieve/pii/S0025619618300727 
3. Terré JA, George I, Smith CR. Pros and cons of transcatheter aortic valve implantation (TAVI). Ann Cardiothorac Surg [Internet]. 2017 Sep;6(5):444-52. Available from: http://www.annalscts.com/article/view/16397

4. Cahill TJ, Chen M, Hayashida K, Latib A, Modine T, Piazza N, et al. Transcatheter aortic valve implantation: current status and future perspectives. Eur Heart J [Internet]. 2018 Apr 27;39(28):2625-34. Available from: https://doi.org/10.1093/ eurhearti/ehy244

5. Allende R, Urena M, Cordoba JG, Ribeiro HB, Amat-Santos I, DeLarochellière R, et al. Impact of the Use of Transradial Versus Transfemoral Approach as Secondary Access in Transcatheter Aortic Valve Implantation Procedures. Am J Cardiol [Internet]. 2014 Dec 1 [cited 2019 Dec 31];114(11):1729-34. Available from: https://www.sciencedirect.com/science/ article/pii/S0002914914017846

6. Bonaros N, Petzina R, Cocchieri R, Jagielak D, Aiello M, Lapeze J, et al. Transaortic transcatheter aortic valve implantation as a first-line choice or as a last resort? An analysis based on the ROUTE registry†. Eur J Cardio-Thoracic Surg [Internet]. 2017 Mar 28;51(5):919-26. Available from: https://doi.org/10.1093/ejcts/ezw406

7. Pierard L. Transcatheter aortic valve implantation: indications. E-Journal Cardiol Pract [Internet]. 2016 [cited 2019 Dec 31];14(1). Available from: https://www.escardio.org/Journals/E-Journal-of-Cardiology-Practice/Volume-14/transcatheter-aortic-valve-implantation-indications

8. Koifman E, Magalhaes M, Kiramijyan S, Escarcega RO, Didier R, Torguson R, et al. Impact of transfemoral versus transapical access on mortality among patients with severe aortic stenosis undergoing transcatheter aortic valve replacement. Cardiovasc Revascularization Med [Internet]. 2016;17(5):318-21. Available from: http://www.sciencedirect.com/science/article/pii/S1553838916301154

9. Modine T, Lemesle G, Azzaoui R, Sudre A. Aortic valve implantation with the CoreValve ReValving System via left carotid artery access: First case report. J Thorac Cardiovasc Surg [Internet]. 2010 Oct;140(4):928-9. Available from: https://doi. org/10.1016/i.jtcvs.2010.03.001\%0A

10. Mylotte D, Sudre A, Teiger E, Obadia JF, Lee M, Spence M, et al. Transcarotid Transcatheter Aortic Valve Replacement: Feasibility and Safety. JACC Cardiovasc Interv [Internet]. 2016;9(5):472-80. Available from: http://www.sciencedirect. com/science/article/pii/S1936879815019251

11. Wee IJY, Stonier T, Harrison M, Choong AMTL. Transcarotid transcatheter aortic valve implantation: A systematic review. J Cardiol [Internet]. 2018;71(6):525-33. Available from: http://www.sciencedirect.com/science/article/pii/ $\underline{\mathrm{S} 0914508718300297}$

12. Overtchouk P, Alqdeimat I, Coisne A, Fattouch K, Modine T. Transcarotid approach for TAVI: an optimal alternative to the transfemoral gold standard. Ann Cardiothorac Surg [Internet]. 2017 Sep;6(5):555-7. Available from: http://www. annalscts.com/article/view/16391/16543

13. Nombela-Franco L, Webb J, de Jaegere P, Toggweiler S, Nuis R-J, Dager A, et al. Timing, Predictive Factors, and Prognostic Value of Cerebrovascular Events in a Large Cohort of Patients Undergoing Transcatheter Aortic Valve Implantation. Circulation [Internet]. 2012 Dec 18;126(25):3041-53. Available from: https://doi.org/10.1161/CIRCULATIONAHA.112.110981 